Canad. Math. Bull. Vol. 19 (3), 1976

\title{
WEAK PARALLELOGRAM LAWS FOR BANACH SPACES
}

\author{
W. L. BYNUM ${ }^{(1)}$
}

It has been shown previously that the $L_{p}(\mu)$ spaces for $1<p \leq 2$ satisfy a weak parallelogram law, and the same methods can be used to show that the $L_{p}(\mu)$ spaces for $2 \leq p<\infty$ satisfy a related weak parallelogram law. This paper obtains several equivalent characterizations of Banach spaces which satisfy one of these two weak parallelogram laws. One such characterization involves the conditions on the moduli of convexity and smoothness analyzed by Lindenstrauss.

1. Introduction. This paper is an outgrowth of the following two theorems. Theorem 1 was established in [1] and Theorem 2 is a new result. Its proof is quite like that of Theorem 1 and need not be given here. As noted in [1], the proof of Theorem 1 (and thus of Theorem 2) is valid for an abstract $L_{p}(\mu)$ space with at least two disjoint sets of positive finite measure.

Theorem 1. If $1<p \leq 2$ and $0<b \leq p-1$, then for all $x, y \in \ell_{p}$,

$$
\|x+y\|^{2}+b\|x-y\|^{2} \leq 2\|x\|^{2}+2\|y\|^{2} ;
$$

moreover, $p-1$ is the largest number $b$ satisfying inequality (1) for all $x$ and $y$ in $\ell_{p}$.

THEOREM 2. If $2 \leq p<\infty$ and $p-1 \leq b<\infty$, then inequality (1) is reversed for all $x, y \in \ell_{p}$; moreover, $p-1$ is the smallest number $b$ such that inequality (1) is reversed for all $x$ and $y$ in $\ell_{p}$.

It is well-known and easy to show that a Banach space satisfying inequality (1) (or the reverse of inequality (1)) for $b=1$ must satisfy the parallelogram identity and by the paper of Jordan and von Neumann [6] must be an inner product space. Inequality (1) for $0<b<1$ and the reverse of inequality (1) for $1<b<\infty$ are two ways to weaken the well-known parallelogram identity. This paper characterizes Banach spaces which satisfy one of these two weak parallelogram laws.

The following terminology will be used.

Definitions. A Banach space $V$ is a lower weak parallelogram space with constant $b$ (or briefly $V$ is $L W P(b)$ ) if inequality (1) if satisfied for each $x$ and $y$

Received by the editors October 8, 1975 and, in revised form, March 25, 1976.

${ }^{(1)}$ Research supported by a Faculty Research Grant of the College of William and Mary. 
in $V . V$ satisfies a lower weak parallelogram law on its sphere with constant $b$ (or briefly $V$ is $L W P S(b)$ ) if inequality (1) holds for all $x$ and $y$ of norm one. $V$ is an upper weak parallelogram space with constant $b$ (or briefly $V$ is $U W P(b)$ ) is inequality (1) is reversed for each $x$ and $y$ in $V$, and finally $V$ is $U W P S(b)$ is inequality (1) is reversed for all $x$ and $y$ of norm one. If $V$ is $\operatorname{LWP}(b)$ (resp. UWP $(b)$ ) for some value of $b$, the best LWP (resp. UWP) constant for $V$ is defined to be $\max \{b: V$ is $\operatorname{LWP}(b)\}($ resp. $\min \{b: V$ is UWP $(b)\})$. The best LWP (or UWP) constant for the unit sphere of $V$ is defined similarly.

Throughout this paper, $V$ will denote a Banach space of dimension greater than one with real scalars and $V^{*}$ will denote its dual.

The definitions imply that any Banach space is $\operatorname{LWP}(b)$ for $b \leq 0$, and if $V$ is UWP $(b)$ then $b \geq 0$. For these reasons all WP constants are assumed to be non-negative. Note also that if $V$ is $\operatorname{LWPS}(b)$ or $\operatorname{LWP}(b)$ then $b \leq 1$, and if $V$ is $\operatorname{UWPS}(b)$ or UWP $(b)$ then $b \geq 1$.

The following observation will be useful in what follows. The substitutions $u=x+y$ and $v=x-y$ in (1) show that $V$ is $\operatorname{LWP}(b)$ if and only if for each $u, v \in V$,

$$
2\|u\|^{2}+2 b\|v\|^{2} \leq\|u+v\|^{2}+\|u-v\|^{2},
$$

and $V$ is $\operatorname{UWP}(b)$ if and only if inequality $\left(1^{\prime}\right)$ is reversed for all $u, v \in V$.

This paper obtains several alternate characterizations of LWP and UWP space. Theorem 3 of section 2 characterizes WP spaces in terms of the normalized duality mapping of the space. Theorems 4 and 5 of section 3 show that if a WP law holds on the unit sphere of a Banach space, then the entire spaces satisfies a WP law of the same type. Although the proofs of Theorems 4 and 5 are too crude to show in general that the best WP constant for the unit sphere is equal to the best WP constant for the whole space, this more precise result is obtained in Theorem 6 for the $L_{p}(\mu)$ spaces for $1<\mathrm{p}<\infty$. Theorems 7 and 8 of section 4 show that the LWP and UWP laws are equivalent, respectively, to the conditions on the moduli of convexity and smoothness analyzed by Lindenstrauss in [7], and these results are used in Theorem 9 to obtain a duality between LWP and UWP spaces.

\section{Normalized duality mapping.}

Definition (cf. [3]). For a Banach space $V$, the normalized duality mapping of $V$ into $V^{*}$ is a map $J: V \rightarrow 2^{V^{*}}$ such that for each $x \in V$,

$$
J x=\left\{f \in V^{*}: f(x)=(f, x)=\|x\|^{2}=\|f\|^{2}\right\} .
$$

The characterization of a WP space in terms of its normalized duality mapping is as follows:

THEOREM 3. Let $V$ be a Banach space with normalized duality mapping $J$. 
Then:

(A) $V$ is $L W P(b)$ if and only if for each $x, y \in V$ and $f \in J x$,

$$
\|x+y\|^{2} \geq\|x\|^{2}+b\|y\|^{2}+2(f, y) \text {. }
$$

(B) $V$ is $U W P(b)$ if and only if for each $x, y \in V$ and $f \in J x$, inequality (2) is reversed.

Proof. To prove part (A), suppose that $V$ is $\operatorname{LWP}(b)$. For $x, y \in V$ and each integer $n \geq 1$, we shall first prove the following inequality:

$$
\|x+y\|^{2}-\|x\|^{2} \geq b\left(1-2^{-n}\right)\|y\|^{2}+2^{n}\left(\left\|x+2^{-n} y\right\|^{2}-\|x\|^{2}\right) .
$$

Indeed, note that the LWP law applied to $(x+y) / 2$ and $x / 2$ yields the case $n=1$, which, when combined with mathematical induction, establishes the desired result for $n>1$.

If $a_{n}$ denotes the right-most term of inequality (3) and if $f \in J x$, then because $f /\|x\|$ has norm one, $\left\|x+2^{-n} y\right\| \geq\|x\|^{-1} f\left(x+2^{-n} y\right)$, and so

$$
a_{n} \geq 2(f, y)+2^{-n}\|x\|^{-2}(f, y)^{2},
$$

from which inequality (2) follows. The reverse implication part (A) is obvious.

To prove part $(B)$, assume that $V$ is $\operatorname{UWP}(b)$. This implies that inequality (3) is reversed for all $x, y \in V$ and $n \geq 1$. If $a_{n}$ is defined as before, then

$$
a_{n} \leq 2^{1-n} b\|y\|^{2}+2^{n}\left(\|x\|^{2}-\left\|x-2^{-n} y\right\|^{2}\right),
$$

by the reverse of inequality ( $\left.1^{\prime}\right)$. For $f \in J x$, the right-most term of inequality (5) is not greater than the right side of inequality (4), and this establishes the desired result.

By replacing $y$ with $-(x+y)$, Theorem 3 can be restated as follows:

Theorem 3'. Let $V$ be a Banach space with normalized duality mapping $J$. Then:

(A) $V$ is $L W P(b)$ if and only if for each $x, y \in V$ and $f \in J x$,

$$
b\|x+y\|^{2} \leq\|x\|^{2}+\|y\|^{2}+2(f, y) .
$$

(B) $V$ is $U W P(b)$ if and only if for each $x, y \in V$ and $f \in J x$, the inequality of part $(\mathrm{A})$ is reversed.

3. Weak parallelogram laws on the unit sphere. It seems natural to expect a Banach space satisfying a WP law on its unit sphere to be a WP space, and the next two theorems support this expectation.

Theorem 4. If a Banach space $V$ is $\operatorname{LWPS}\left(b^{2}\right)$, then $V$ is $\operatorname{LWP}(b /(1+b))^{2}$.

Proof. Assume the contrary; i.e., that $V$ is $\operatorname{LWPS}\left(b^{2}\right)$ and $V$ is not $\operatorname{LWP}\left(c^{2}\right)$ 
where $c=b /(1+b)$. Then there must exist $u, v \in V$ such that $\|u\|=1 \leq\|v\|$ and

$$
\|u+v\|^{2}+c^{2}\|u-v\|^{2}>2+2\|v\|^{2} .
$$

Thus by the triangle inequality $c\|u-v\| \geq\|v\|-1$, and if $w=v /\|v\|$ then $\|u-w\| \geq(1+b)^{-1}\|u-v\|$. Therefore,

$$
\begin{aligned}
4 & \geq\|u+w\|^{2}+b^{2}\|u-w\|^{2} \\
& \geq\|u+v\|^{2}-2\left(\|v\|^{2}-1\right)+(\|v\|-1)^{2}+c^{2}\|u-v\|^{2} \\
& >4+(\|v\|-1)^{2},
\end{aligned}
$$

which is contradictory.

Theorem 5. If a Banach space $V$ is UWPS $(b)$, then $V$ is $U W P(4 b+1)$.

Proof. From the assumption that $V$ is not $\operatorname{UWP}(4 b+1)$, there exist $u, v \in V$ which violate the UWP law with constant $4 b+1$ and satisfy $\|u\|=1 \geq\|v\|$. The remainder of the proof is similar to the proof of Theorem 4 and is omitted.

With regard to the well-known parallelogram law and the unit sphere, Day [2] has shown that if a Banach space satisfies the parallelogram law on its unit sphere, then the parallelogram law holds for all of $V$; or stated in the terminology of this paper, if $V$ is both LWPS(1) and UWPS(1), then $V$ is LWP(1). Schoenberg [8] later improved Day's result to show that if $V$ is LWPS(1) (or UWPS(1)) then $V$ is LWP(1). These results suggest the conjecture that if $V$ is $\operatorname{LWPS}(b)$ for $0<\mathrm{b}<1$ (or $V$ is UPWS $(b)$ for $1<b<\infty$ ) then $V$ is $\operatorname{LWP}(b)$ (or $V$ is $\operatorname{UWP}(b)$ ), or equivalently stated, the best WP constant for the unit sphere of $V$ is equal to the best WP constant of $V$.

The argument given for the above theorems is too crude to establish this result; in the above argument, too much was given up to escape from the unit sphere. It is not clear whether the previous conjecture is true in general, but it does hold in the particular case of the $L_{p}(\mu)$ spaces for $1<p<\infty$.

THEOREM 6. For $1<p \leq 2, \max \left\{b: L_{p}(\mu)\right.$ is $\left.\operatorname{LWPS}(b)\right\}=p-1$, and for $2 \leq$ $p<\infty, \min \left\{b: L_{p}(\mu)\right.$ is $\left.U W P S(b)\right\}=p-1$.

Proof. Case $1.1<p \leq 2$. Note that for a Banach space $V$ the best LWP constant for the unit sphere of $V$ is

$$
\inf \left\{\left(4-\|x+y\|^{2}\right) /\|x-y\|^{2}:\|x\|=\|y\|=1, x \neq y\right\} .
$$

Denote this number by $A_{V}$. If $\delta_{V}(t)$ is the modulus of convexity of $V$ (see section 4 for a definition), then we shall show in what follows that:

$$
A_{V}=\inf \left\{4\left(1-\left(1-\delta_{V}(t)\right)^{2}\right) / t^{2}: 0<t \leq 2\right\} \text {. }
$$

Denote the number on the right side of equation (6) by $B_{V}$. For any $x, y \in V$ 
such that $\|x\|=\|y\|=1$ and $\|x-y\|=t>0$, it follows from the definition of $\delta_{V}(t)$ that

$$
4\left(\left(1-\left(1-\delta_{V}(t)\right)^{2}\right) \leq 4-\|x+y\|^{2}\right.
$$

and therefore $B_{V} \leq A_{V}$. Now pick $0<t \leq 2$ and $\varepsilon>0$. Select $x, y \in V$ with norm one such that $\|x-y\|=t$ and $2-\|x+y\| \leq 2 \delta_{V}(t)+2 \varepsilon t^{2}$. Then,

$$
4-\|x+y\|^{2} \leq 4\left(\delta_{V}(t)+\varepsilon t^{2}\right)\left(2-\delta_{V}(t)\right)
$$

and so,

$$
A_{V} \leq 4\left(1-\left(1-\delta_{V}(t)\right)^{2}\right) / t^{2}+8 \varepsilon .
$$

Therefore $A_{V} \leq B_{V}$, and equation (6) is established.

In the particular case of $V=L_{p}(\mu)$ for $1<p \leq 2$, Hanner [5, Theorem 2] has shown that for $0 \leq t \leq 2, \delta_{V}(t)$ is the unique solution of the equation:

$$
\left(1-\delta_{V}(t)+(t / 2)\right)^{p}+\left|1-\delta_{V}(t)-(t / 2)\right|^{p}=2 .
$$

It follows from (6) and (7) that $A_{V}$ is the infimum of the set

$$
\left\{\left(1-u^{2}\right) / v^{2}: 0<v<1,(u+v)^{p}+|u-v|^{p}=2\right\},
$$

so that for $0<b<a$ and $a^{p}+b^{p}=2$,

$$
A_{V} \leq\left(4-(a+b)^{2}\right) /(a-b)^{2}
$$

Two applications of L'Hôpital's rule show that as $a \downarrow 1$ the expression on the right approaches $p-1$, and hence $A_{V} \leq p-1$. Since Theorem 1 yields the opposite inequality, the proof is complete.

Case 2. $2 \leq p<\infty$. For a Banach space $V$ and for $0 \leq t \leq 2$, define $\gamma_{V}(t)$ to be

$$
\sup \{1-(\|x+y\| / 2):\|x\|=\|y\|=1,\|x-y\|=t\} .
$$

As in Case 1, the best UWP constant for the unit sphere of $V$ turns out to be

$$
\sup \left\{4\left(1-\left(1-\gamma_{V}(t)\right)^{2} / t^{2}: 0<t \leq 2\right\} .\right.
$$

A repetition of Hanner's argument for Theorem 2 of [5] shows that when $V=L_{p}(\mu)$ for $2 \leq p<\infty, \gamma_{V}(t)$ is the unique solution of the equation obtained from (7) by replacing $\delta_{V}(t)$ with $\gamma_{V}(t)$. The remainder of the proof of Case 2 is similar to the proof of Case 1 and is omitted.

It is mildly surprising that the trick used in [1] to show that $p-1$ is the best LWP constant for $\ell_{p}(1<p \leq 2)$ does not work here to prove Case 1 of Theorem 6. In [1], $y_{t}$ is defined to be $(1+t) e_{1}+(1-t) e_{2}$ where $e_{1}$ and $e_{2}$ are the first two unit coordinate vectors in $\ell_{p}$, and the limit of $\left(2\left\|y_{0}\right\|^{2}+2\left\|y_{t}\right\|^{2}-\| y_{0}+\right.$ $\left.y_{t} \|^{2}\right) /\left\|y_{0}-y_{t}\right\|^{2}$ as $t \rightarrow 0$ is shown to be $p-1$. However, if $y_{0}$ and $y_{t}$ are replaced by $y_{0} /\left\|y_{0}\right\|$ and $y_{t} /\left\|y_{t}\right\|$, the limit as $t \rightarrow 0$ turns out to be $2(p-1) /(3-p)$ which is larger than $p-1$. 
4. The convexity and smoothness conditions of Lindenstrauss. Duality. It turns out that a UWP law (or LWP law) is equivalent to a smoothness (or convexity) condition investigated by Lindenstrauss in [7]. These conditions are given below. First, recall that the modulus of convexity of a Banach space $V$ is a function, $\delta_{V}(t)$, defined for $t$ in $[0,2]$ to be:

$$
\inf \{1-(\|x+y\| / 2):\|x\|=\|y\|=1,\|x-y\|=t\}
$$

and the modulus of smoothness of $V$ is a function, $\rho_{V}(t)$, defined for $t \geq 0$ to be:

$$
\left(\frac{1}{2}\right) \sup \{\|x+t y\|+\|x-t y\|-2:\|x\|=\|y\|=1\} .
$$

Recall also that $V$ is uniformly convex if $\delta_{V}(t)>0$ for $0<t \leq 2$, and $V$ is uniformly smooth if the right derivative of $\rho_{V}$ at 0 is 0 .

Definition. A Banach space $V$ is said to satisfy a Lindenstrauss convexity condition with constant $b$ (or briefly, $V$ is $L C(b)$ ) if $b$ is a positive number such that for each $t$ in $[0,2] \delta_{V}(t) \geq b t^{2}$. A Banach space $V$ is said to satisfy a Lindenstrauss smoothness condition with constant $b$ (or briefly, $V$ is $L S(b)$ ) if for each $t \geq 0 \rho_{V}(t) \leq b t^{2}$.

It is clear from the definitions that an LC space is uniformly convex and that an LS space is uniformly smooth.

The next two theorems establish the equivalence of LC and LWP spaces and of LS and UWP spaces.

THEOREM 7. (A) If a Banach space $V$ is $L C\left(b^{2}\right)$, then $V$ is $L W P(2 b /(1+2 b))$.

(B) If $V$ is $L W P(b)$, then $V$ is $L C(b / \AA)$.

Proof. The proof follows immediately from equation (6) and Theorem 3.

THEOREM 8. (A) If a Banach space $V$ is $L S(b)$, then $V$ is $U W P\left(1+4 b+2 b^{2}\right)$. (B) If $V$ is $U W P(b)$, then $V$ is $L S(b / 2)$.

Proof. For $x, y \in V$ of norm one and $0 \leq t \leq 1$, let $c=\|x+t y\|$ and $d=$ $\|x-t y\|$.

To prove (A), note that $V$ is $\operatorname{LS}(b)$, then

$$
c^{2}+d^{2}+2 c d \leq 4+\left(8 b+4 b^{2}\right) t^{2} .
$$

If $f \in J x$, then $1-t^{2} \leq 1-t^{2}(f, y)^{2} \leq c d$, from which (A) follows.

To prove $(\mathrm{B})$, note that

$$
c+d-2 \leq\left(\frac{1}{4}\right)\left((c+d)^{2}-4\right) \leq\left(\frac{1}{2}\right)\left(c^{2}+d^{2}-2\right) .
$$

If $V$ is $\operatorname{UWP}(b)$, then the last expression is not greater than $b^{2}$, which establishes part (B).

A reasonable conjecture is that if $V$ is both UWP and LWP, then $V$ is isomorphic (linearly homeomorphic) to a Hilbert space, and this is indeed true. 
In fact, Figiel and Pisier in [4] have shown much more; namely, if $V$ has an equivalent LC (or LWP) norm and an equivalent LS (or UWP) norm, then $V$ is isomorphic to a Hilbert space.

Lindenstrauss has already shown in [7] that LC and LS spaces are dual. In particular, using Theorem 1 of [7] and Theorems 7 and 8 above, we obtain:

THEOREM 9. Let $V$ be a Banach space.

(A) If $V$ is $L W P(b)$, then $V^{*}$ is $U W P\left(1+2 b^{-1}+2^{-1} b^{-2}\right)$.

(B) If $V$ is $U W P(b)$, then $V^{*}$ is $L W P\left(1+(b / 2)^{1 / 2}\right)^{-2}$.

There are two facts which indicate that the WP constants in Theorem 9 can perhaps be improved. First, for $1<p<\infty$ the WP constant for $L_{p}(\mu)$ is $p-1$ while the WP constant for $L_{p}(\mu)^{*}$ is $1 /(p-1)$ (for $\mu \sigma$-finite). Second, using the normalized duality mapping, one can show that if $V$ is $\operatorname{LWP}(b)$ then $V^{*}$ is UWP $(1+16 / b)$, and this UWP constant is smaller than the UWP constant of Theorem $9(\mathrm{~A})$ when $0<b<\frac{1}{28}$.

\section{REFERENCES}

1. W. L. Bynum and J. H. Drew, A weak parallelogram law for $\ell_{p}$, Amer. Math. Monthly 79 (1972), 1012-1015.

2. M. M. Day, Some characterizations of inner product spaces, Trans. Amer. Math. Soc. 62 (1947), 320-337.

3. C. R. DePrima and W. V. Petryshyn, Remarks on strict monotonicity and surjectivity properties of duality mappings defined on real normed linear spaces, Math. Z. 123 (1971), 49-55.

4. T. Figiel and G. Pisier, Séries aléatoires dans les espaces uniformément convexes ou uniformḿent lisses, C. R. Acad. Sci. Paris Ser. A 279 (1974), 611-614.

5. O. Hanner, On the uniform convexity of $L^{p}$ and $\ell^{p}$, Ark. Mat. 3 (1956), 239-244.

6. P. Jordan and J. von Neumann, On inner products in linear metric spaces, Ann. of Math. (2) 36 (1935), 719-723.

7. J. Lindenstrauss, On the modulus of smoothness and divergent series in Banach spaces, Michigan Math. J. 10 (1963), 241-252.

8. I. J. Schoenberg, A remark on M. M. Day's characterization of inner product spaces and a conjecture of L. M. Blumenthal, Proc. Amer. Math. Soc. 3 (1952), 961-964.

College of William and Mary

WiLLIAMSBURG, ViRginia 23185

U.S.A. 